\title{
ARQUEOLOGÍA ESTRATIGRÁFICA Y RESTAURACIÓN
}

\author{
(STRATIGRAPHIC ARCHAEOLOGY AND RESTORATION)
}

G. P. Brogiolo, Arquitecto

Fecha de recepción: 13-II-95

Dpto. de Arqueología e Historia del Arte

, Univ. de Siena

ITALIA

RESUMEN

Tras el rápido desarrollo de la estratigrafia arqueológica, ocurrida a partir de los años -70 , pronto se consideró esencial e insustituible su aplicación automática al análisis de la construcción histórica. Esto dió lugar de inmediato a una reacción crítica que dudaba de la rígida aplicación del análisis estratigráfico al edificio, ya que dejaba sin resolver los problemas de interacción, teóricos y prácticos, entre los campos de la historia de la arquitectura, de su conocimiento técnico y cientifico y de la actividad restauradora. En este texto se analiza la relación entre arqueología estratigráfica, historia de la arquitectura y restauración, buscando los puntos comunes entre estas disciplinas y la ayuda que la primera supone para el desarrollo de sus compañeras.

\section{SUMMARY}

After the rapid development of the archaeological stratigraphy, which began in the 70s, its automatic application in the analysis of the historical constructions propmtly became considered as essential and irreplaceable. This immediately gave rise to a critical reaction which questioned the rigid application of the stratigraphic analysis to the building, since it left unsolved the theoretical and practical problems of interaction between the fields of history, architecture, its technical and scientific knowledge, and restoration activity. This text analyzes the relation between the stratiraphic archaeology, the history of architecture and the restoration, looking for common points between these disciplines, and underscoring the help the former provides in the development of the latter ones.
La arqueología estratigráfica ha constituido, desde fines del decenio de 1970 y el comienzo del siguiente, una formidable oleada que ha puesto en cuestión las teorías y métodos de las ciencias arqueológicas, arraigadas desde mucho tiempo atrás.

Los estratígrafos llegaron a teorizar sobre el valor absoluto del método, ya que en la primera parte del estudio ... no hay necesidad de tener en cuenta para nada la importancia histórica de los diversos estratos (Harris 1983, p. 79), con la consecuencia de que se puede aplicar a la construcción como a cualquier depósito arqueológico enterrado, sin ninguna especificación ni adecuación (Manacorda 1985; Parenti 1985, 1988).

En seguida, como toda fuerza revolucionaria en su fase de movimiento, se ha dedicado a conquistar nuevos territorios, en particular los administrados, con un aire decadente de
Ancien Régime -tanto en el plano de los métodos como en el de los resultados- por la historia de la arquitectura y las disciplinas enlazadas con la restauración arquitectónica.

A la vez que se abrían grandes huecos en las defensas de estas consolidadas disciplinas, que dejaban pasar a las vanguardias triunfantes de la estratigrafia consideradas esenciales e insustituibles de modo casi universal, se planteaba la resolución, en el plano teórico, de la dicotomía (a veces en el límite de la conflictividad, Bonelli 1986) que se iba creando entre la nueva disciplina y

a) la historia de la arquitectura;

b) los otros instrumentos del conocimiento científicotécnico; $y$

c) los aspectos teóricos y prácticos te la restauración arquitectónica. 
En tal sentido se manifestaron a fines de los años -80 (Doglioni 1987, 1988; Brogiolo 1988) las primeras voces críticas que subrayaban que la complejidad y la riqueza de la información inscrita en la estratigrafía de un edificio eran tales que no se podían captar con los esquemas conceptualmente rígidos de la arqueología estratigráfica.

\section{Arqueología estratigráfica e historia de la arquitectura}

Un edifício no está constituido sólo por estratos, sino también por formas. La lectura estratigráfica, al limitarse a documentar y secuenciar las acciones constructivas, no posee los instrumentos conceptuales que definen los aspectos estilísticos y formales de un edificio.

Es insustituible para reconocer los acontecimientos constructivos, pero no sabe explicar su significado. Éste lo aporta el bagaje teórico de la historia del arte que, actuando por esquemas analógicos, reconstruye los aspectos formales de un edificio y permite completar la investigación historiando la aséptica secuencia conseguida por la arqueología estratigráfica.

El diagrama-representación de la secuencia de las unidades estratigráficas o acciones constructivas- se podrá de este modo completar con la identificación de los elementos estructurales y formales -huecos, ornamentación, elementos estructurales estáticos-, que no se hacen evidentes en la lectura estratigráfica por estar insertos en acciones constructivas más generales (Fig. 1).
Los conocimientos histórico-artísticos son también indispensables para establecer la equivalenciaentre distintas acciones constructivas, operación ésta que permite ordenar la secuencia y reconocer las llamadas interfaces de periodo o conjunto de las unidades estratigráficas que en una fase determinada definen la forma de un edificio.

Por tanto el análisis no puede llevarse a cabo por escaladores solitarios. Ni por el estratígrafo, yaque su bagaje conceptual prescinde de los aspectos histórico-formales, ni tampoco por el historiador de la arquitectura, pues su disciplina no ha teorizado la identificación de la unidad estratigráfica ni los modos para establecer sus relaciones.

Por ahora podemos suponer la coexistencia de dos caminos, el estratigráfico y el histórico-artístico. Pero debemos favorecer que ambos puedan ser recorridos por una misma persona: un arqueólogo que conozca la historia de la arquitectura 0 , mejor, un historiador de la arquitectura que haya asimilado los instrumentos conceptuales de la arqueología estratigráfica.

\section{Arqueología estratigráfica y restauración}

A diferencia de la excavación, el análisis estratigráfico de edificios tiene un límite muy preciso: no se puede desmontar un edificio entero, una unidad estratigráfica tras otra, para comprender mejor las relaciones entre los estratos.

Incluso en un edificio en curso de restauración, existen límites objetivos y límites éticos. Los primerosson evidentes:
Fig.1.- Este esquema resume el doble recorrido lógico del "análisis estratigráfico" (a, identificación de las unidades estratigráficas; $b$, identificación de las relaciones; c, construcción de un diagrama) y del análisis formal o histórico - artistico (a, identificación de los elementos formales o unidad de referencia; $b$, de las relaciones analógicas). Los dos recorridos paralelos permiten periodizar la secuencia.
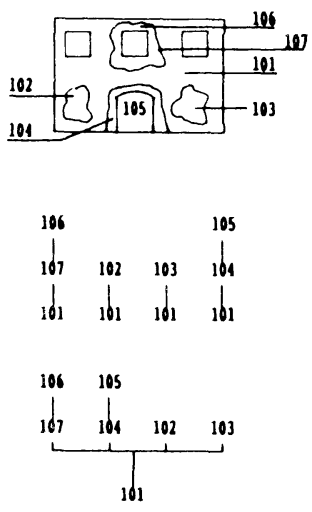
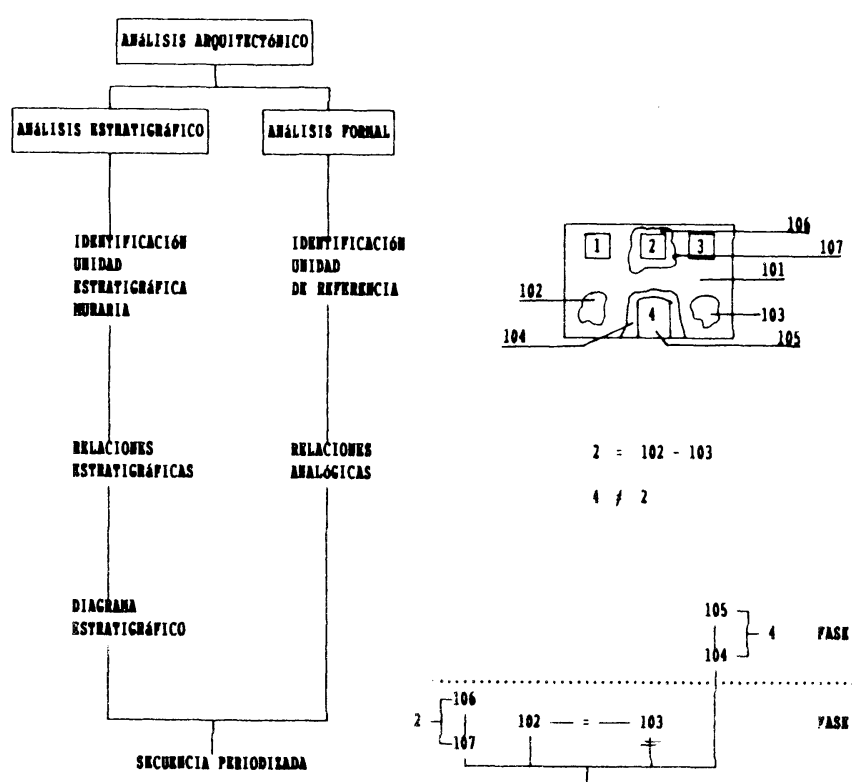

$$
104
$$

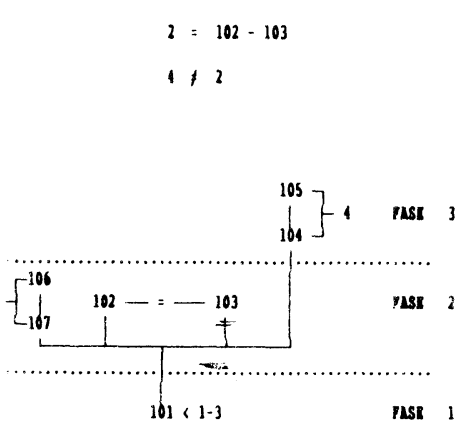


sobre los segundos hay que efectuar una reflexión que implica a las propias opciones de la restauración-sobre las que se vuelve al final de este texto-

Un edificio está hecho, además de la fábrica misma, de películas -enlucidos, cielos rasos, tabiques adosados-, una serie de envoltorios, también ellos estratificados, que esconden las fases constructivas más antiguas.

Una teoría de la restauración, que en Italia ha tenido en los últimos años una presencia académica relevante-Bellini y Dezzi Bardeschi por ejemplo-, sostiene que la restauración debe reducirse a conservar lo existente. Las fases más antiguas únicamente se pueden documentar con análisis diagnósticos no destructivos -por ejemplo mediante termografia o georradar-.

Técnicas que, por otra parte, restituyen una imagen muy desenfocada de estas fases más antiguas, ya que no son capaces de ir más allá de una confirmación genérica de las discontinuidades del tejido de la obra.

Estos ensayos tienen, por otrolado, solamente un significado cognoscitivo y no están encaminados a definir la restauración, ya que se ha decidido a priori no modificar en nada el aspecto adquirido por el edificio. Se rechaza un juicio de valor, por lo que se acepta, por ejemplo, que en el Barco della Regina Cornaro de Altivole, un palacio principesco renacentista en la alta llanura véneta, los establos, realizados en el s. XX, deshacen la imagen tardo cuatrocentista que tiene el complejo.
Se trata de una postura extrema que valora del mismo modo las superposiciones-incidentales en el sentido etimológico del término- que han destruido las fases constructivas intervenciones más generales y consabidas-. Es decir, que se contenta con observar la portada del libro-edificio, a menudo sin relación alguna con todo lo que se esconde en los muros, sin deshojarlo para leer su historia.

Si esta teoría de la restauración se generalizase, quedaría muy poco espacio para el estudio estratigráfico. Afortunadamente existen otras y entonces se tiene la objetiva necesidad de desnudar la fábrica de los añadidos que la revisten para intervenir sobre el equilibrio estructural.

Operación esta última en la que, en presencia de una ruina, la lectura estratigráfica es indispensable. La elección de muestras a analizar no se puede confiar al acaso, sino que ha de hacerse con premeditación sobre la base de una secuencia estratigráfica.

Durante la restauración del siglo pasado de la iglesia de Sta. Sofía de Padua, un insigne monumento bizantinorománico de los ss. XI-XII construido con ladrillos sesquipedales, se fabricaron ladrillos de las mismas dimensiones y características que los antiguos, pero cuyas respuestas físicas son bastante distintas. Un muestreo que no hubiese tenido en cuenta la secuencia no habría comprendido los diferentes resultados provocados por los materiales empleados en los ss. XI y XII respecto a los de las restauraciones. El mismo problema se plantea en el castillo de Valbona, donde largos lienzos de la muralla son

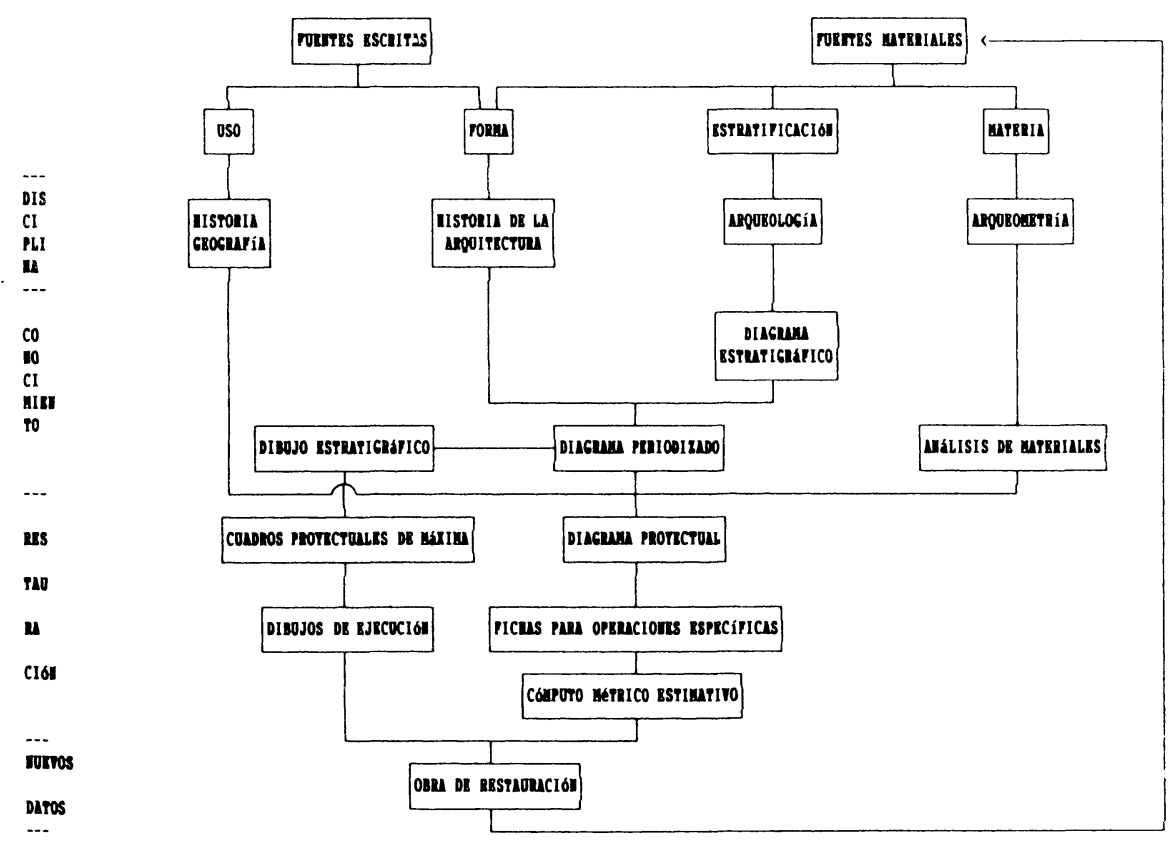

Fig.2.- Del conocimiento al proyecto de restauración. El esquema resume los caminos cognoscitivos de distintas disciplinas -historia, geografia humana, historia de la arquitectura, arqueología, arqueometria-que, analizando los diversos aspectos de un edificio -función, forma, estratificación, material-, producen informaciones útiles para las opciones de restauración. Éstas, a su vez, se pueden puntualizar en un diagrama de proyecto e ilustrarse con detalle en la documentación unida al proyecto. La obra de restauración, al sacar a la luz etementos nuevos no observables anteriormente, reactiva el proceso cognoscitivo. 
obra de restauración imitativa (Figs. 2 y 3).

Más allá aún de este empleo secundario, se le reconoce a la arqueología estratigráfica haberse situado en una posición central respectoa todas las hipótesis de restauración que no se proponen el embalsamamiento de lo existente, incluidas las telarañas y las grietas del enlucido. Sobre todo por aquéllas, bastante discutibles, que proponen la utilización del edificio histórico para contener nuevas figuras arquitectónicas, salvando su envoltura externa pero destruyendo la secuencia interior. Práctica que ha tenido ejemplos ilustres como el de Carlo Scarpa en el museo de Castelvecchio de Verona e imitadores mucho más modestos -o desmañados- en una práctica arquitectónica hoy universalmente difundida en Italia que conserva el aspecto formal exterior de los edificios de los centros históricos, transformándolo interiormente en el estilo de la propiedad horizontal.

Frente a estas opciones destructivas, la arqueología estratigráfica al menos genera conocimiento histórico, trasladando al plano documental lo que ya no existe materialmente.

Por el contrario, es en las opciones que se proponen subrayar la entidad histórica de un edifício donde la arqueología estratigráfica no es solamente un instrumento de conocimiento, sino que se hace clave insustituible del proceso de restauración.

Son opciones que prevén el sacrificio de algunas porciones recientes de la secuencia de un edificio para permitir la lectura de las fases más antiguas, de modo que el edificio ostente materialmente toda su historia, no una sola fase: ni laactual, como en las líneas teóricas que se han mencionado antes; ni la original, opción que, tras la estela de Viollet le Duc, ha visto florecer en Italia, entre fines del s. XIX y la primera mitad de éste, castillos medievales, iglesias románicas, centros históricos de mil doscientos y mil trescientos, sujetos arquitectónicos fascinantes que todavía hoy atraen manadas de turistas, pero casi siempre falsificados.

Lo que no significa por otra parte proponer un patchwork, que pretenda documentar cada una de las mínimas transformaciones realizadas. Es el diagrama periodizado el que, al convertirse en diagrama de proyecto (Brogiolo 1993), nos ha de guiar en la elección de qué cosas deben mostrarse (Fig. 4).

Se forma, en primer lugar, una continuidad lógica entre la fase cognoscitiva -arqueología e historia artística sintetizadas en el diagrama-, las opciones iniciales del proyecto y las correcciones impuestas por las nuevas informaciones obtenidas al ejecutarse la obra de restauración. Lo que presupone una estrecha colaboración

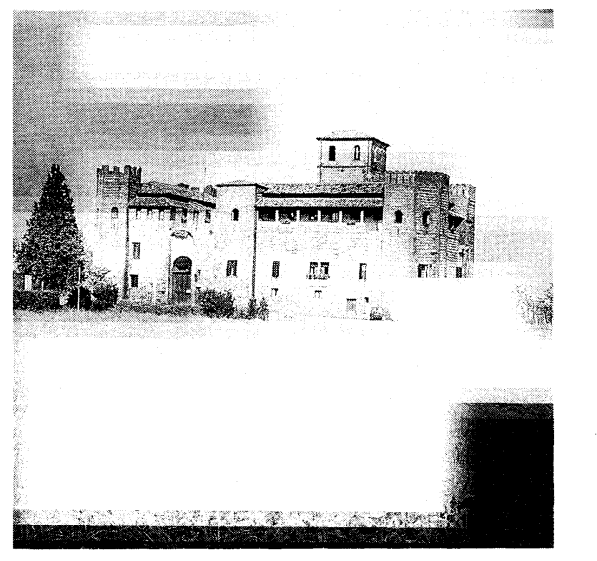

Fig.3.- Castillo de Valbona (Padua).

entre quienes generan conocimiento y quienes ejercen las decisiones del proyecto.

Las decisiones del proyecto se podrán efectuar de modo consciente sobre la base de las interfaces de periodo momentos unitarios y sucesivos de la construcción que se han citado anteriormente-. Algunas interfaces se considerarán más significativas -está implícito un criterio de valoración- y por tanto se subrayarán con el proyecto de restauración. Otras, juzgadas menos relevantes -como los establos del 1900 del Barco della Regina Cornaro-, se podrán eliminar o atenuar. Se logra de este modo una compenetración eficaz de la autoconciencia del proyectista, que será capaz de valorar el impacto de su intervención sobre el peso histórico del edificio. Por ejemplo, en el proyecto de restauración de la fachada de la Academia Carrara de Bérgamo (análisis estratigráfico y consultoría del autor y de Luca Zigrino en un proyecto de Vittorio Gregotti), se decidió suprimir casi totalmente la fase decimonónica -conversión en cuartel-, para destacar una fase del s. XIII -edificio con tabernas en planta baja (Fig. 5)- y otra renacentista -establecimiento de un complejo monástico-.

Tampocovamos a olvidar algunas posibilidades secundarias de un proyecto anclado en el diagrama estratigráfico:

1) posibilidad, cuando sea necesario, de puntualizar las intervenciones operativas referentes a una unidad estratigráfica particularmente significativa;

2) guía detallada para el encargado de obra;

3) control eficaz e inequívoco para los servicios de inspección;

4) oportuna aprobación de los trabajos ejecutados al finalizar la obra. 


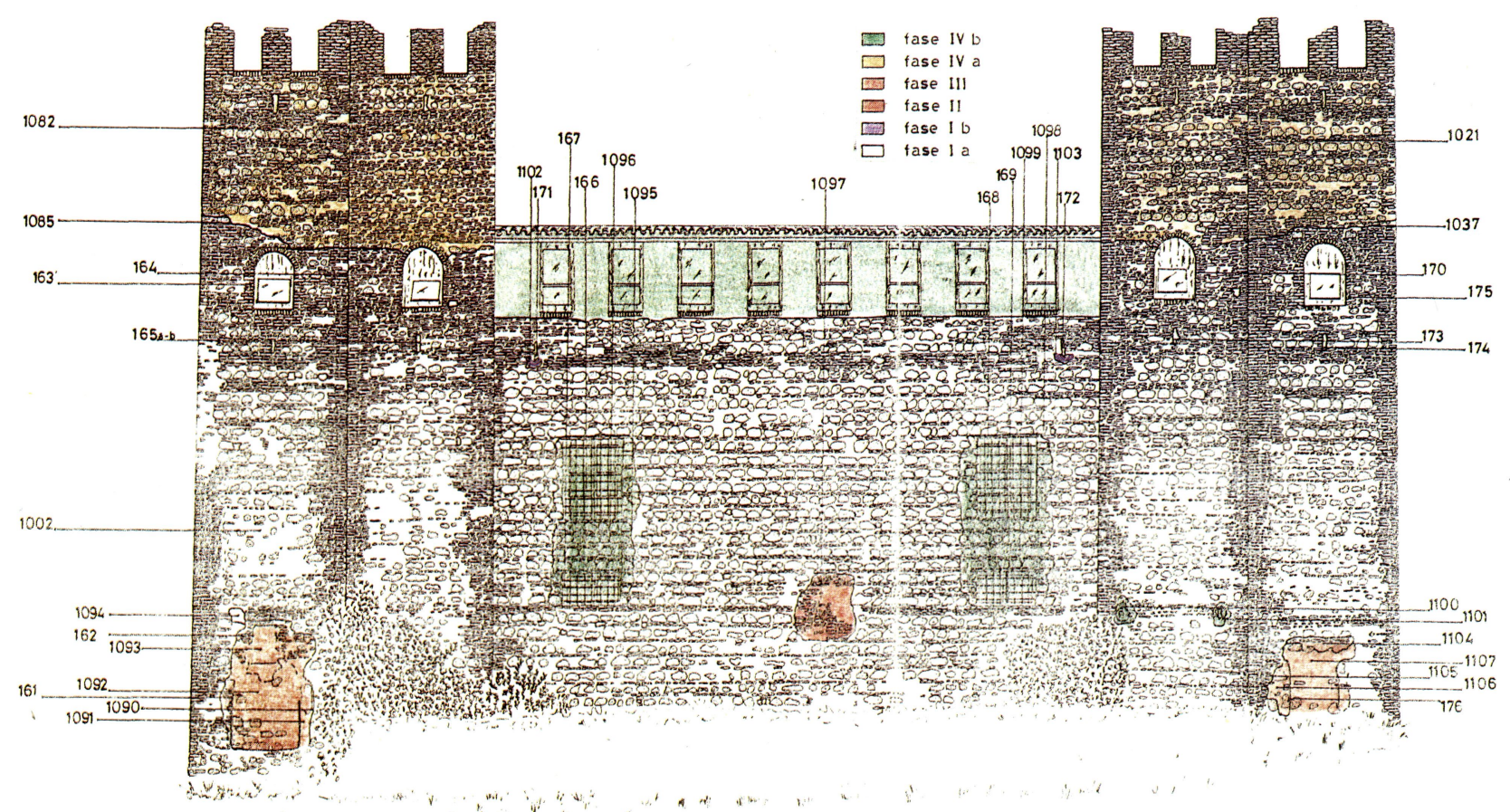

Fig.4.- Castillo de Valbona (Padua), cara S. Análisis estratigráfico: la fase IV (a,b) corresponde a intervenciones de restauración y modificaciones recientes.

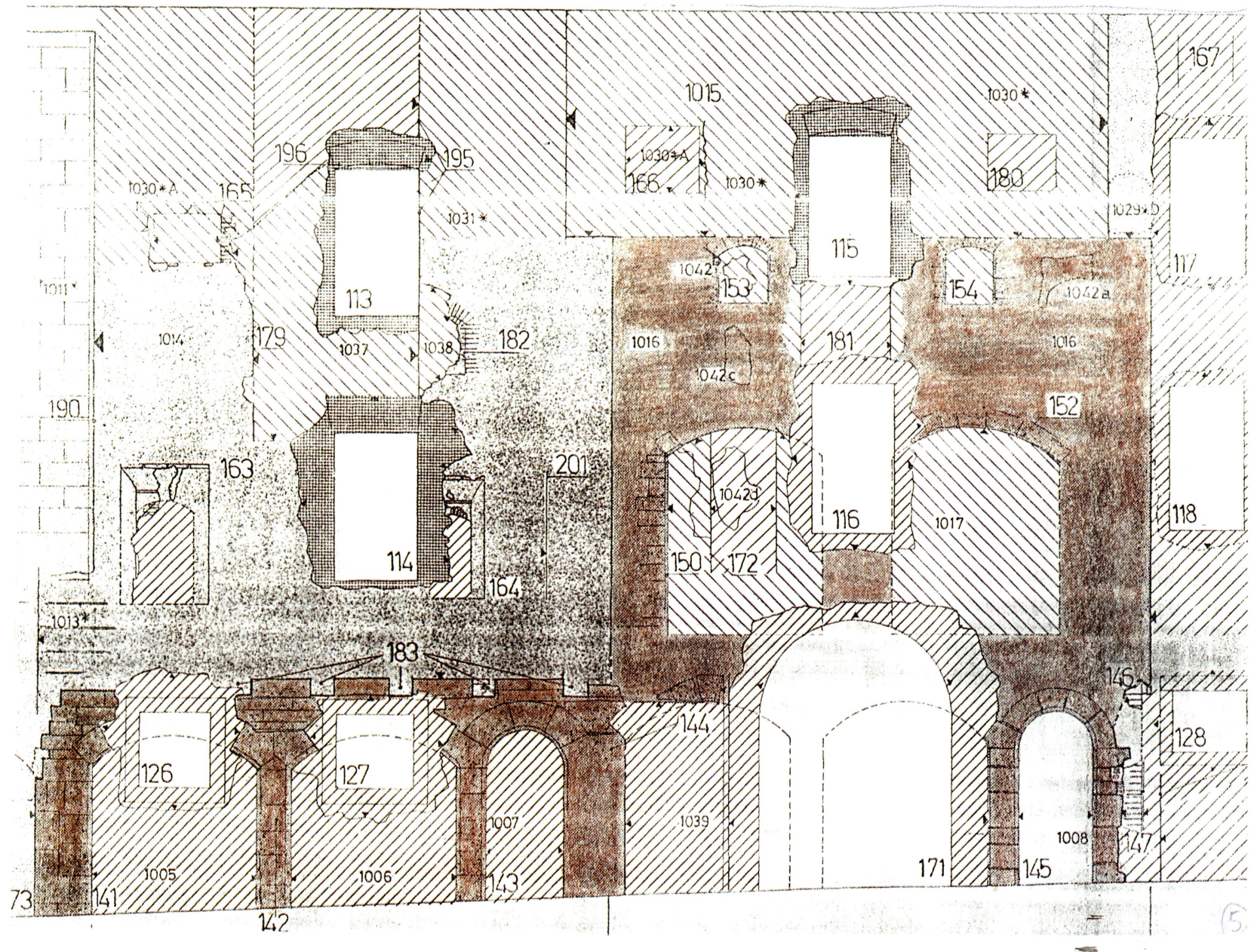

Fig.5.- Bérgamo, Academia Carrara. Análisis estratigráfico evidenciando la fase de fines del s. XIII que se destacó en el proyecto de restauración (Brogiolo y Zigrino, para un proyecto de Vittorio Gregotti). 
BONELLI R. 1986, Archeologia stratigrafica e Storia dell 'architettura, Storia e documenti, 2, pp. 5-10.

BROGIOLOG. P. 1988, Archeologia dell 'edilizia storica, Como.

BROGIOLO G. P. 1993, Appunti su analisi stratigrafica e restauro, en M. Uboldi (editor) Carta archeologica della Lombardia. III. Como. La città murata e la convalle, Modena 1993, pp. 102-108.

DOGLIONI F. 1987 (editor), Ambienti di dimore medievali a Verona, Verona.

DOGLIONI F. 1988, La ricerca sulle strutture edilizie tra archeologia stratigrafica e restauro architettonico, en Francovich, Parenti 1988, pp. 223-246.
FRANCOVICH R., Parenti R. 1988, Archeologia e restauro di monumenti, Florencia.

HARRIS E. C. 1983, Principi di stratigrafia archeologica, Roma, traducción italiana e introducción de D. Mancorda, Florencia.

MANACORDA D. 1985, Appunti su archeologia e architettura nel cantiere della crypta Balbi, Restauro e Città, I, n. 2, pp. 2132 .

PARENTI R. 1985, La lettura stratigrafica delle murature in contesti archeologici e di restauro architettonico, Restauro e Città, I, n.2, pp. 55-68.

PARENTI R. 1988, Le techniche di documentazione per una lettura stratigrafica dell 'elevato, en Francovich, Parenti 1988, pp. 249-279.

\section{Publicación del Instituto Eduardo Torroja - CSIC}

\section{Número monográfico de INFORMES}

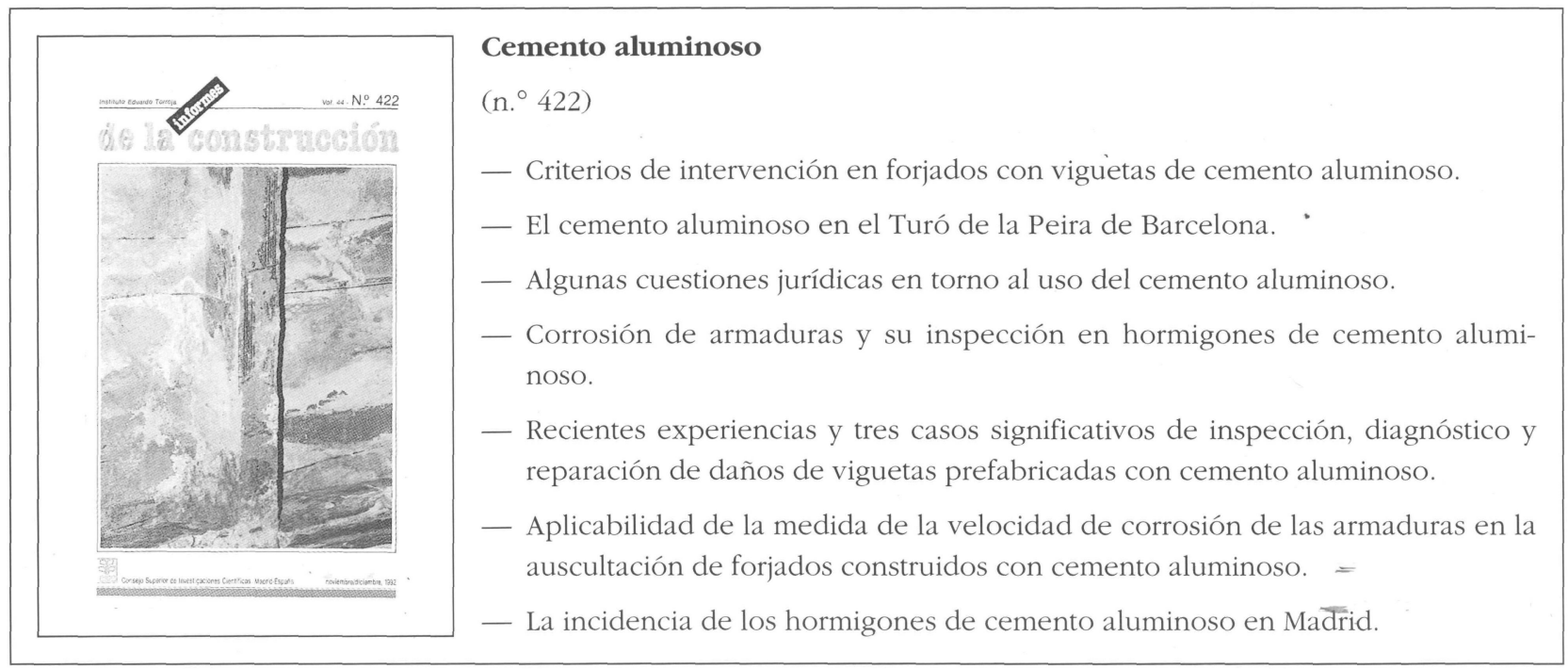

